\title{
Evaluation du besoin minimum d'entretien en phosphore chez l'agneau*
}

\author{
F Meschy, L Guéguen
}

Station de Recherches de Nutrition, INRA, 78350 Jouy-en-Josas, France

\begin{abstract}
Summary - The purpose of the present study was to determine the minimum level of endogenous fecal loss of $P$ in sheep receiving a $P$-deficient and Ca-rich diet composed of natural feedstuffs. The low fecal endogenous loss of $P(15 \mathrm{mg} / \mathrm{kg} / \mathrm{d})$ was obtained under abnormal physiological conditions and did not correspond to the minimum requirement for maintenance.
\end{abstract}

Introduction - L'évaluation de la part incompressible de la perte fécale endogène de $P$, correspondant au besoin minimal d'entretien, est toujours sujette à controverses. Ainsi, cette perte minimale est de $25 \mathrm{mg} / \mathrm{kg} / \mathrm{j}$ pour. I'INRA (INRA, 1978; Guéguen et al, 1988) et de $12 \mathrm{mg} / \mathrm{kg} / \mathrm{j}$ seulement pour l'ARC (1980). Cependant, cette dernière valeur, qui correspond à l'extrapolation théorique à un ingéré nul de $P$, est maintenant contestée par les auteurs anglais eux-mêmes (Todd, 1984; Braithwaite, 1985).

L'objectif de la présente étude est de déterminer le niveau minimum d'excrétion fécale endogène de $P$ chez l'agneau recevant un régime à base d'aliments naturels carencé en $\mathrm{P}$ et riche en calcium.

Matériel et Méthodes - Deux lots de 6 agneaux mâles castrés de race lle de France d'un poids moyen de $28 \mathrm{~kg}$ en début d'expérience reçoivent l'un des deux régimes expérimentaux: normal en P (NP: $4,3 \mathrm{~g} / \mathrm{kg} \mathrm{MS}$ ) ou carencé (BP: $1,0 \mathrm{~g} \mathrm{P} / \mathrm{kg} \mathrm{MS}$ ), tous deux à base d'aliments naturels (pulpes de betteraves, manioc, paille traitée par la soude). Après 3 semaines d'adaptation, un bilan apparent est effectué sur $\mathrm{P}, \mathrm{Ca}, \mathrm{Mg}$ et cellulose; $\mathrm{P}, \mathrm{Ca}$ et $\mathrm{Mg}$ sont également dosés dans le plasma. A la suite de ces bilans et après une injection souscutanée de $37 \mathrm{MBq}$ de ${ }^{32} \mathrm{P}$, la mesure du phosphore fécal endogène est réalisée par la méthode de dilution isotopique.

Résultats et Discussion - La perte fécale endogène est élevée pour le lot NP, mais l'ingéré est également élevé et il est désormais bien connu qu'au delà de l'entretien strict la perte fécale endogène en $P$ croît avec le niveau alimentaire; en revanche, la faible valeur pour le lot $B P$, néanmoins supérieure à celle retenue par I'ARC (1980), conduit à des perturbations physiologiques importantes : hypophosphatémie marquée, chutes de l'ingestion $(-25 \%)$ et de la croissance, faible digestibilité de la cellulose pour le lot BP.

Malgré un large excès relatif de calcium (Ca/P de 16 pour le lot BP), la digestibilité réelle de $P$ n'est pas significativement diminuée bien qu'elle soit, pour chacun des lots, notablement plus faible que les résultats obtenus avec des régimes semi-synthétiques où $P$ est apporté sous forme de phosphates minéraux de bonne qualité nutritionnelle (Boxe-

\footnotetext{
* Communication aux IVes Journées des Recherches sur l'Alimentation de la Nutrition des Herbivores de mars 1988, non publié par erreur en 1989
} 
Tableau I. Effets de la carence en phosphore sur le métabolisme de P.

\begin{tabular}{lccc}
\hline & $N P$ & $B P$ & $P$ \\
\hline $\begin{array}{lccc}\text { Ingéré (g/j) } \\
\text { Fécal endogène } \\
\text { (mg/j/kg) }\end{array}$ & 4,05 & 0,67 & $<0,001$ \\
Absorption réelle (\%) & 48,0 & 15,1 & $<0,001$ \\
Retenu (g/) & 62,0 & 52,2 & NS \\
Phosphatémie (mg/l) & 1,03 & $-0,08$ & $<0,001$ \\
& 76,7 & 27,7 & $<0,001$ \\
\hline
\end{tabular}

beld et al, 1983). La différence de digestibilité réelle observée entre les deux lots peut être attribuée à la part du phosphate bicalcique contenu dans le régime NP $(1,55 \%$ de MS).

En conclusion, cette expérience montre que le besoin minimum d'entretien (croissance exclue) en phosphore chez le mouton est nettement supérieur à $15 \mathrm{mg}$ par $\mathrm{kg}$ et par jour et que toute valeur inférieure conduit à une forte sous-estimation du besoin dans des conditions usuelles d'élevage. La perte endogène de $P$ de $25 \mathrm{mg} /$ $\mathrm{kg} / \mathrm{j}$ retenue par l'INRA (1978) est vraisemblable dans la mesure où l'on vise des conditions physiologiques et alimentaires normales et une bonne minéralisation osseuse. Dans ces conditions, on peut estimer le besoin en $P$ de l'agneau à $1,4 \mathrm{~g} / \mathrm{j}$ pour l'entretien strict et à $3,2 \mathrm{~g} / \mathrm{j}$ avec un objectif de croissance de $200 \mathrm{~g} / \mathrm{j}$.

Agricultural Research Council (1980) Livestock. Commonwealth Agricultural Bureaux Slough, UK

Boxebeld A, Guéguen L, Hannequart G, Durand M (1983) Reprod Nutr Dév 23, 1043-1053

Braithwaite GD (1983) J Agric Sci Cambridge $105,67-72$

Guéguen L, Durand $M$, Meschy $F$ (1988) Bull Tech CRZV Theix, INRA 70, 105-112

INRA (1978) Alimentation des Ruminants. INRA, Versailles

Todd JR (1984) Recent Advances in Animal Nutrition. Butterworths, London 